\title{
Criminologie
}

\section{Échelle auto-rapportée de psychopathie de Levenson : adaptation française et validation}

\section{Claudia Savard, Yvan Lussier et Stéphane Sabourin}

Volume 47, numéro 2, automne 2014

URI : https://id.erudit.org/iderudit/1026736ar

DOI : https://doi.org/10.7202/1026736ar

Aller au sommaire du numéro

\section{Éditeur(s)}

Les Presses de l’Université de Montréal

ISSN

0316-0041 (imprimé)

1492-1367 (numérique)

Découvrir la revue

Citer cet article

Savard, C., Lussier, Y. \& Sabourin, S. (2014). Échelle auto-rapportée de psychopathie de Levenson : adaptation française et validation. Criminologie, 47(2), 263-293. https://doi.org/10.7202/1026736ar
Résumé de l'article

L'échelle auto-rapportée de psychopathie de Levenson (LSRP ; Levenson, Kiehl \& Fitzpatrick, 1995) est un instrument visant à évaluer les traits de personnalité psychopathiques au sein de la population générale. Il conceptualise la psychopathie en deux facteurs distincts, mais interreliés, appelés psychopathie primaire et secondaire. La validité de l'instrument dans son format original est bien démontrée, mais aucune donnée n'est disponible en ce qui a trait à l'adaptation francophone de l'instrument. L'étude vise donc à évaluer la structure factorielle et la validité convergente de la version française auprès d'une population de jeunes adultes. Un échantillon de 2529 participants âgés de 15 à 26 ans remplissent l'échelle auto-rapportée de psychopathie, le NEO-FFI et répondent aussi à des questions sur leurs habitudes à l'égard de la consommation de substances psychoactives. Les résultats des analyses factorielles exploratoires et confirmatoires montrent que l'adaptation francophone respecte la structure en deux facteurs, avec des coefficients de saturation et de consistance interne pour les deux échelles se comparant à ceux de la version originale anglaise. Les modèles de mesure pour les hommes et les femmes se sont avérés partiellement invariants ; les hommes obtiennent des résultats supérieurs à ceux des femmes aux échelles de psychopathie primaire et totale. Enfin, le LSRP démontre de bons indices de validité convergente. 


\title{
Échelle auto-rapportée de psychopathie de Levenson: adaptation française et validation
}

\author{
Claudia Savard ${ }^{1}$ \\ Professeure adjointe \\ Département des fondements et pratiques en éducation, Université Laval \\ claudia.savard@fse.ulaval.ca \\ Yvan Lussier \\ Professeur titulaire \\ Département de psychologie, Université du Québec à Trois-Rivières \\ yvan.lussier@uqtr.ca \\ Stéphane Sabourin \\ Professeur titulaire \\ École de psychologie, Université Laval \\ stephane.sabourin@psy.ulaval.ca
}

RÉSUMÉ • L'échelle auto-rapportée de psychopathie de Levenson (LSRP; Levenson, Kiehl \& Fitzpatrick, 1995) est un instrument visant à évaluer les traits de personnalité psychopathiques au sein de la population générale. Il conceptualise la psychopathie en deux facteurs distincts, mais interreliés, appelés psychopathie primaire et secondaire. La validité de l'instrument dans son format original est bien démontrée, mais aucune donnée n'est disponible en ce qui a trait à l'adaptation francophone de l'instrument. L'étude vise donc à évaluer la structure factorielle et la validité convergente de la version française auprès d'une population de jeunes adultes. Un échantillon de 2529 participants âgés de 15 à 26 ans remplissent l'échelle auto-rapportée de psychopathie, le NEO-FFI et répondent aussi à des questions sur leurs habitudes à l'égard de la consommation de substances psychoactives. Les résultats des analyses factorielles exploratoires et confirmatoires montrent que l'adaptation francophone respecte la structure en deux facteurs, avec des coefficients de saturation et de consistance interne pour les deux échelles se comparant à ceux de la version originale anglaise. Les modèles de mesure pour les hommes et les femmes se sont avérés partiellement invariants; les hommes

1. Université Laval, Pavillon des Sciences de l'éducation, Faculté des sciences de l'éducation, Département des fondements et pratiques en éducation, Québec, (Québec), Canada, G1V 0A6. 
obtiennent des résultats supérieurs à ceux des femmes aux échelles de psychopathie primaire et totale. Enfin, le LSRP démontre de bons indices de validité convergente.

MOTS-CLÉs • Psychopathie, validité, analyses factorielles, personnalité, consommation de substances psychoactives.

\section{Introduction}

La psychopathie est un concept élaboré par Cleckley (1976) pour dépeindre des individus présentant une variété de comportements antisociaux et qui entretiennent des relations intimes teintées d'exploitation et de manipulation. La conceptualisation théorique contemporaine décrit la psychopathie comme une constellation de traits de personnalité communément regroupés sous deux facteurs distincts, mais interreliés (Brinkley, Newman, Widiger \& Lynam, 2004; Hare, 1991; Harpur et al., 1988, 1989; Karpman, 1948). Le premier facteur, nommé psychopathie primaire, considéré par Karpman (1948) comme étant la «vraie psychopathie», fait référence à la sphère interpersonnelleémotionnelle du construit et est caractérisé par des individus affichant une attitude narcissique, cynique, manipulatrice, égoïste et insensible, prêts à utiliser sans scrupules le mensonge et le charme superficiel pour parvenir à leurs fins. À cela s'ajoute une très faible capacité à ressentir des remords et de l'anxiété. Le second facteur, appelé psychopathie secondaire, fait davantage référence à la sphère comportementale du construit et décrit des individus souvent impulsifs et anxieux (Benning, Patrick, Hicks, Blonigen \& Krueger, 2003), qui sont incapables de planifier à long terme, de tolérer les frustrations et qui présentent de fortes tendances antisociales. Reconnu au départ auprès de populations carcérales, le concept de psychopathie a aussi été employé pour comprendre le fonctionnement psychosocial au sein de populations infracliniques (Forth, Brown, Hart \& Hare, 1996; Lilienfeld \& Andrews, 1996; Lynam, Whiteside \& Jones, 1999; Salekin, Trobst \& Krioukova, 2001; Widiger, 1998). D’ailleurs, plusieurs chercheurs ont établi la présence de traits de personnalité psychopathiques infracliniques au sein de la population générale (Brinkley et al., 2004; Savard, Sabourin $\&$ Lussier, 2006, 2011). Leur apparition peut survenir dès l'enfance ou l'adolescence et ils demeureraient stables jusqu'à l'âge adulte (Lynam et al., 2005 ; Salekin, Rosenbaum \& Lee, 2012), entraînant de nombreuses conséquences. Dans des contextes occupationnels, ces traits de personnalité sont associés à des vols, de la supervision abusive, un leadership 
malsain, une prise de décision non éthique et à de l'intimidation en milieu de travail (Babiak \& Hare, 2007; Buss, 1993; Caponecchia, Sun \& Wyatt, 2012; Hall \& Benning, 2006; O’Boyle, Forsyth, Banks \& McDaniel, 2012; Poon, 2003; Stevens, Deuling \& Armenakis, 2012; Tepper, 2007). En ce qui a trait aux relations conjugales, quelques données documentent des degrés importants d'insatisfaction conjugale et sexuelle (Jonason \& Buss, 2012; Kastner \& Sellbom, 2012; Savard et al., 2006, 2011), la présence d'infidélité (Egan \& Angus, 2004) ainsi que de la violence conjugale et sexuelle (Holtzworth-Munroe, Meehan, Herron, Rehaman \& Stuart, 2003) au sein des couples où l'un des conjoints présente ces traits de personnalité. Les taux de prévalence des traits psychopathiques répertoriés au sein de la population générale oscillent entre $2 \%$ et $13 \%$ (p. ex. Hare, 1993; Levenson et al., 1995 ; Savard et al., 2006, 2011).

L'Échelle auto-rapportée de psychopathie de Levenson (LSRP; Levenson et al., 1995; traduction française par Sabourin \& Lussier, 1998) est un questionnaire constitué de 26 items mesurant les traits de personnalité psychopathiques au sein de la population générale. Les deux facteurs discutés précédemment sont reproduits dans les analyses factorielles de l'instrument (Brinkley, Schmitt, Smith \& Newman, 2001; Levenson et al., 1995; Lynam et al., 1999; Savard, Lussier, Sabourin \& Brassard, 2005). Plusieurs études sur la validité de l'instrument démontrent que: (a) celui-ci présente une bonne validité convergente et divergente (Brinkley et al., 2001; Lynam et al., 1999; Hill, Neumann \& Rogers, 2004); les deux échelles corrèlent significativement avec la sensibilité à l'ennui et la désinhibition (Levenson et al., 1995); (b) l'échelle secondaire est associée à la présence d'anxiété (Levenson et al., 1995); (c) le résultat total au questionnaire de psychopathie est positivement associé à la consommation d'une grande variété de drogues, d'alcool et de comportements antisociaux ainsi qu'à une plus grande probabilité d'avoir connu des démêlés avec la justice au moins une fois dans sa vie (Blonigen, Carlson, Hicks, Krueger \& Iacono, 2008; Lee \& Salekin, 2010; Strand \& Belfrage, 2005); (d) des études portant sur la théorie en cinq facteurs associent négativement l'échelle primaire au facteur Amabilité, et l'échelle secondaire serait aussi négativement associée aux facteurs Amabilité et Sens des responsabilités, alors qu'elle serait positivement liée au facteur Névrosisme (Lynam et al., 1999; Widiger, 1998); (e) il semble que les gens affichant des traits de personnalité psychopathiques infracliniques présenteraient 
les mêmes déficits dans la modulation des réponses affectives que les psychopathes incarcérés (Lynam et al., 1999); et (f) finalement, la présence de traits psychopathiques globaux et secondaires chez les hommes serait significativement reliée à leur détresse conjugale et à celle de leur conjointe sur une période d'un an, à une prédominance de styles d'attachement évitant ainsi qu'à l'usage de violence psychologique en relation (Savard et al., 2004, 2006).

En dépit de ces résultats, différents auteurs ont remis en question la structure en deux facteurs à partir de résultats issus d'études sur la validation de l'instrument (Brinkley, Diamon, Magaletta \& Heigel, 2008; Sellbom, 2011). Il semble que plusieurs items de l'instrument discriminent peu entre les degrés de psychopathie chez divers groupes de participants (hommes incarcérés, femmes incarcérées, étudiants universitaires; Sellbom, 2011). En plus, des réserves ont également été émises concernant les différences sexuelles quant à la sévérité des traits présentés, mais également à l'expression de ceux-ci (Marion \& Sellbom, 2012). Bref, les questionnements suscités dans la littérature invitent à la réalisation d'études de validation ayant recours à une variété d'échantillons provenant de la population générale.

\section{Objectif}

L'objectif de la présente étude est d'évaluer les qualités psychométriques de la version française de l'échelle auto-rapportée de psychopathie de Levenson auprès d'un large échantillon d'adolescents et de jeunes adultes québécois provenant de la population générale. L'analyse des propriétés métriques de cet instrument auprès d'adolescents et de jeunes adultes constitue une priorité de recherche puisque les spécialistes croient généralement que les traits psychopathiques se développent relativement tôt (Ribeiro da Silva, Rijo \& Salekin, 2013; Salekin \& Lynam, 2010). En conséquence, il est important de démontrer que les qualités du LSRP s'appliquent aussi à ce type d'échantillon. La consistance interne, l'association de chacun des items à son facteur latent et la structure factorielle de l'instrument seront documentées. L'étude vise aussi à évaluer s'il existe une différence significative entre les hommes et les femmes quant au modèle de mesure de la psychopathie et quant aux résultats obtenus au LSRP. Enfin, une analyse de validité convergente est également présentée. Celle-ci sera menée à l'aide d'un instrument évaluant les traits de personnalité selon le modèle en cinq facteurs 
et d'items portant sur l'attitude à l'égard de la consommation de substances psychoactives. Ces deux éléments de validité de convergence sont privilégiés puisqu'ils permettent d'une part de reproduire les résultats obtenus avec la version originale anglaise du LSRP et parce qu'ils peuvent être utilisés auprès d'une population infraclinique. En dépit de ces avantages et de son statut de mesure étalon, nous n'avons pas jugé opportun ici de faire usage du Psychopathic Checklist-Revised (Hare, 2003). Il s'agit d'un instrument destiné de prime abord à une clientèle carcérale et qui requiert généralement la présence du dossier judiciaire pour être coté, ce que nous avions peu de chances d'obtenir auprès d'un échantillon d'adolescents et de jeunes adultes.

\section{Méthode}

\section{Participants}

L'échantillon est constitué de 2529 participants âgés de 15 à 26 ans de niveau secondaire et collégial (852 hommes et 1677 femmes). Les participants ont été recrutés dans diverses écoles secondaires et établissements d'enseignement collégial de la région de la Mauricie et de Québec sur une base volontaire. À la suite des explications données en classe sur le projet, les participants intéressés ont rempli un formulaire de consentement et se sont vu remettre une enveloppe contenant des questionnaires. Une fois remplis, ceux-ci devaient être retournés par la poste. Les femmes de l'échantillon sont âgées en moyenne de 17,82 ans (É.T. $=1,44$ an), possèdent en moyenne 11,5 années de scolarité et un revenu annuel moyen de 5399,13 \$CAN (É.T. = 4092,41 \$CAN). Les hommes sont âgés en moyenne de 17,63 ans (É.T. $=1,54$ an), possèdent en moyenne 11,27 années de scolarité et un revenu annuel moyen de 6242,59 \$ CAN (É.T. = 6184,75 \$ CAN).

\section{Instruments}

Les participants ont rempli l'échelle auto-rapportée de psychopathie de Levenson (LSRP; Levenson et al., 1995; adaptation française par Sabourin \& Lussier, 1998; Annexe A). L'instrument est composé de 26 items évaluant les traits psychopathiques dans la population générale selon les dimensions retrouvées dans le Hare Psychopathic Checklist (PCL-R; Hare, 1991), soit la psychopathie primaire et secondaire. Les 
auteurs ont généré les items de façon à minimiser les biais associés à la désirabilité sociale. La réponse à chaque item est cotée selon une échelle de type Likert en quatre points allant de «Fortement en désaccord» à «Fortement en accord». Les coefficients alpha obtenus dans les études canadiennes et américaines varient de 0,77 à 0,85 pour l'échelle totale, de 0,76 à 0,83 pour l'échelle de psychopathie primaire et de 0,59 à 0,69 pour l'échelle secondaire (Brinkley et al., 2001; Levenson et al., 1995; Savard et al., 2005, 2006). Les deux échelles du LSRP sont modérément corrélées ( $\mathrm{r}=0,43$ à 0,46; Lynam et al., 1999; Miller et al., 2008) et significativement associées aux deux facteurs du PCL-R (Brinkley et al., 2001). Les deux échelles sont également corrélées à des mesures d'abus de substances et de criminalité. Les coefficients alpha obtenus dans la présente étude sont de 0,77 pour l'échelle totale, de 0,78 pour l'échelle de psychopathie primaire et de 0,63 pour l'échelle de psychopathie secondaire, ce qui est somme toute comparable à ce qui a été obtenu dans la version originale. Dans la présente étude, la relation entre les deux facteurs latents s'est également avérée significative $(r=0,40)$.

Le NEO-FFI (Costa \& McCrae, 1992; traduction française par Sabourin \& Lussier, 1992) est une version brève à 60 items du NEOPersonality Inventory-Revised (NEO-PI-R). Il évalue les traits de la personnalité selon les cinq grands facteurs ou domaines généralement reconnus par la communauté scientifique: Névrosisme (indicateur d'équilibre émotionnel, de sensibilité à la détresse psychologique et de capacité d'adaptation); Extraversion (mesure le degré de sociabilité, d'affirmation, de leadership et d'expression verbale); Amabilité (évalue la bienveillance à l'égard des autres, l'empathie et les tendances interpersonnelles plus intimes); Ouverture à l'expérience (mesure l'ouverture et la souplesse, de même que le regard critique relativement à son expérience); et Sens des responsabilités (évalue le contrôle des impulsions, envies et désirs, ainsi que les capacités de planification et d'organisation). Chaque domaine contient 12 items; les réponses à ces items sont cotées selon une échelle de type Likert à cinq points allant de «Totalement en désaccord» (1) à «Totalement en accord» (5). Les coefficients de consistance interne de la version originale de l'instrument sont adéquats, se situant entre 0,66 (Amabilité) et 0,88 (Névrosisme; Costa \& McCrae, 1992). Finalement, l'instrument démontre de bons indices de validité convergente et discriminante (Costa \& McCrae, 2010). Les coefficients alpha obtenus à chacun des facteurs dans la présente étude sont adéquats et atteignent respectivement 0,83 pour le 
facteur Névrosisme, 0,73 pour l'Extraversion, 0,68 pour l'Ouverture à l'expérience, 0,71 pour l'Amabilité et 0,81 pour le Sens des responsabilités. De nombreux chercheurs se sont penchés, avec un certain succès, sur la capacité de définir les traits de personnalité psychopathiques à l'aide de mesures évaluant la personnalité en cinq facteurs (p. ex. Decuyper, De Clercq, De Bolle \& De Fruyt, 2003; Lee \& Ashton, 2005; Lynam et al., 2005; Paulhus \& Williams, 2002; Salekin et al., 2012; Vachon, Lynam, Widiger, Miller, McCrae \& Costa, 2013). Dans la présente étude, le NEO-FFI, une mesure brève de la personnalité en cinq facteurs, servira donc à évaluer la validité convergente du LSRP.

Les habitudes reliées à la consommation de substances sont évaluées à l'aide de six énoncés, dont trois sur la consommation d'alcool qui sont extraits du Alcohol Use Disorders Identification Test (AUDIT) développé par Saunders, Aasland, Babor, de la Fuente \& Grant, (1993) qui comprend 10 items. Cet instrument a été fréquemment utilisé dans les enquêtes épidémiologiques comme celles de l'Organisation mondiale de la Santé. Nous avons ajouté trois items sur la consommation de drogues en utilisant les mêmes formulations que celles des items sur la consommation d'alcool. Les réponses aux items sont cotées selon une échelle de type Likert en cinq points selon la fréquence et l'intensité des habitudes de consommation. Ils sont regroupés afin de former un indicateur de validité convergente. Un résultat élevé aux six items permet d'identifier les gens présentant une consommation d'alcool et/ ou de drogues problématique avec des conséquences nuisibles sur le fonctionnement. Dans la présente étude, l'analyse des relations entre ces six items montre la présence d'un coefficient de consistance interne s'élevant à 0,67 , ce qui est acceptable compte tenu du nombre limité d'items.

\section{Analyses statistiques}

Les propriétés métriques de l'instrument sont d'abord examinées à l'aide d'une analyse d'items, selon l'approche de la théorie classique des tests: statistiques descriptives des items, corrélations inter-items, item-total (corrélations supérieures à 0,30 ) et coefficient alpha de Cronbach lorsque chaque item est retiré. Des analyses factorielles préliminaires de type exploratoire, effectuées à l'aide du prologiciel statistique SPSS 21.0 ont par la suite été menées sur une moitié de l'échantillon. Des analyses confirmatoires, prenant en considération le mode de 
réponse catégoriel aux items et employant la méthode robuste, ont ensuite été réalisées à l'aide du prologiciel EQS 6.2 sur la seconde moitié de l'échantillon. Les critères utilisés pour évaluer le degré d'ajustement des modèles sont les suivants: (a) des indices CFI (Comparative Fit Index) et NNFI (Non-Normed Fit Index) égaux ou supérieurs à 0,90 (Byrne, 1994; Tabachnik \& Fidell, 2001); (b) un indice RMSEA (Root Mean Square Error of Approximation) égal ou inférieur à 0,05 (Hu \& Bentler, 1999) ou, à la limite, compris entre 0,05 et 0,08 (Hu \& Bentler, 1999; Kline, 2010). Le rapport du chi-carré sur le nombre de degrés de liberté est également examiné, un résultat inférieur à 2 est jugé acceptable alors qu'un résultat inférieur à 5 est jugé appréciable. Par ailleurs, cet indice est considéré comme moins essentiel puisqu'il est reconnu pour être hautement influencé par la taille de l'échantillon. Son application systématique peut mener au rejet de modèles adéquats (Kline, 2010; Tomarken \& Waller, 2003). Par la suite, une série de tests hiérarchiques d'invariance ont été réalisées afin de vérifier si le modèle en deux facteurs obtenu dans l'étude est invariant chez les hommes et les femmes. Les moyennes obtenues par les hommes et les femmes aux échelles de psychopathie totale, primaire et secondaire sont ensuite comparées. Finalement, les analyses de validité convergente sont effectuées à l'aide de corrélations entre le LSRP, d'une part, et les cinq échelles du NEO-FFI ainsi que les items sur les habitudes à l'égard de la consommation de substances psychoactives, d'autre part.

\section{Résultats}

Les résultats moyens obtenus aux échelles de psychopathie, de personnalité et d'habitudes à l'égard de la consommation de substances pour les hommes et les femmes sont présentés dans le Tableau 1. Il est à noter qu'il existe une différence significative entre les hommes et les femmes aux échelles de psychopathie totale et primaire, ce qui est cohérent avec les données issues d'études antérieures (Gummelt, Anestis \& Carbonell, 2012; Levenson et al., 1995; Miller, Gaughan \& Prior, 2008). Des différences significatives ont également été observées entre les hommes et les femmes pour les échelles d'Ouverture à l'expérience ainsi qu'aux items évaluant l'attitude quant à la consommation de substances. Les tailles d'effet pour ces quatre variables, évaluées à l'aide du coefficient $d$ de Cohen, sont respectivement de 0,46, de 0,63, de -0,26 et de -0,82. Lorsque l'on utilise le système de classification proposé par Brinkley et 
al. (2001) pour catégoriser, à l'aide de l'échelle totale, la prévalence de traits psychopathiques chez les individus de l'échantillon, 38,5\% des participants présentent des traits faibles (résultat de moins de 48 à l'échelle totale), 37,9\% rapportent des traits moyens (résultats entre 48 et 58 ) et $23,3 \%$ obtiennent des traits élevés (résultat de plus de 58 ). Le taux de prévalence observé dans l'échantillon peut sembler élevé. Or, il s'agit de traits psychopathiques infracliniques et non de psychopathie avec une forte composante criminelle comme le décrit Hare (1993). Peu de données sont actuellement disponibles pour établir des comparaisons avec le taux de prévalence obtenu dans la présente étude. Par ailleurs, une recherche récente documente, à l'aide d'un instrument différent, des taux de prévalence atteignant $30 \%$ chez une population âgée de moins de 25 ans (Vachon et al., 2013) et diminuant à $20 \%$ après l'âge de 45 ans.

TA B LEA U 1

Statistiques descriptives et comparaisons de moyennes des traits psychopathiques totaux, primaires, secondaires, des cinq traits de personnalité et des habitudes de consommation de substances psychoactives en fonction du sexe des participants

\begin{tabular}{|c|l|l|l|l|l|}
\hline Variables & $\begin{array}{c}\text { Total } \\
\mathrm{N}=2533\end{array}$ & $\begin{array}{c}\text { Hommes } \\
\mathrm{N}=851\end{array}$ & $\begin{array}{c}\text { Femmes } \\
\mathrm{N}=1676\end{array}$ & $F$ & $\begin{array}{c}d \text { de } \\
\text { Cohen }\end{array}$ \\
\hline Psychopathie & & & & & \\
\hline Totale & $\begin{array}{l}51,58 \\
(8,89)\end{array}$ & $\begin{array}{l}54,21 \\
(9,02)\end{array}$ & $\begin{array}{l}50,18 \\
(8,52)\end{array}$ & $6.13^{* *}$ & 0,46 \\
\hline Primaire & $\begin{array}{l}30,69 \\
(6,58)\end{array}$ & $\begin{array}{l}33,36 \\
(6,91)\end{array}$ & $\begin{array}{l}29,29 \\
(6,01)\end{array}$ & $23,19^{* * *}$ & 0,63 \\
\hline Secondaire & $\begin{array}{l}20,69 \\
(4,35)\end{array}$ & $\begin{array}{l}20,59 \\
(4,28)\end{array}$ & $\begin{array}{l}20,56 \\
(4,39)\end{array}$ & 1,05 & 0,007 \\
\hline NE0-FFI & $\begin{array}{l}53,36 \\
(10,26)\end{array}$ & $\begin{array}{l}52,32 \\
(10,41)\end{array}$ & $\begin{array}{l}53,88 \\
(10,14)\end{array}$ & 3,02 & $-0,15$ \\
\hline Névrosisme & $\begin{array}{l}56,21 \\
(10,39)\end{array}$ & $\begin{array}{c}56,31 \\
(10,07)\end{array}$ & $\begin{array}{c}56,15 \\
(10,55)\end{array}$ & 3,00 & 0,016 \\
\hline Extraversion & $\begin{array}{l}49,04 \\
(11,14)\end{array}$ & $\begin{array}{l}47,12 \\
(11,47)\end{array}$ & $\begin{array}{l}50,01 \\
(10,85)\end{array}$ & $4,11^{*}$ & $-0,26$ \\
\hline $\begin{array}{l}\text { Ouverture à } \\
\text { L'expérience }\end{array}$ & $\begin{array}{l}48,18 \\
(11,38)\end{array}$ & $\begin{array}{l}47,64 \\
(11,30)\end{array}$ & $\begin{array}{l}48,46 \\
(11,41)\end{array}$ & 0,02 & $-0,07$ \\
\hline Amabilité & $\begin{array}{l}47,31 \\
(11,63)\end{array}$ & $\begin{array}{l}46,16 \\
(11,29)\end{array}$ & $\begin{array}{l}47,89 \\
(11,76)\end{array}$ & 1,83 & $-0,15$ \\
\hline $\begin{array}{l}\text { Sens des } \\
\text { responsabilités }\end{array}$ & $\begin{array}{c}3,92 \\
(3,00)\end{array}$ & $\begin{array}{l}4,49 \\
(3,36)\end{array}$ & $\begin{array}{c}3,63 \\
(2,75)\end{array}$ & $36,48^{* * *}$ & -0.28 \\
\hline $\begin{array}{l}\text { Habitudes de } \\
\text { consommation }\end{array}$ & & & & \\
\hline
\end{tabular}


${ }^{*} p<0,05 ;{ }^{* *} p<0,01 ;{ }^{* * *} p<0,001$.

\section{Analyse d'items selon la théorie classique des tests}

Les statistiques descriptives pour chacun des items des hommes et des femmes sont rapportées à l'Annexe A. Des différences de moyennes sont observées pour tous les items, à l'exception des items 19, 21 et 26. Les corrélations inter-items varient de $-0,59$ à 0,41 . Des corrélations problématiques (inférieures à 0,10 ou négatives) ont été notées aux items 4 , $10,19,23$ et 26. Lorsque l'on observe les corrélations item-total, les items $4,10,23$ et 26 obtiennent des corrélations inférieures à 0,30 avec le résultat total à l'échelle, faisant ainsi diminuer le coefficient alpha de consistance interne de façon significative. Lorsque l'on retire ces items, le coefficient alpha de l'échelle totale passe de 0,77 à 0,79 , celui de l'échelle de psychopathie primaire passe de 0,78 à 0,80 et celui de l'échelle secondaire passe de 0,63 à 0,64.

\section{Structure factorielle}

Les analyses factorielles exploratoires préliminaires sur les 26 items du questionnaire sont d'abord réalisées sur une moitié de l'échantillon. Elles visent ainsi à reproduire la structure en deux facteurs obtenue par Levenson et ses collaborateurs (1995). Il est à noter qu'il n'y a pas de différence significative entre les deux moitiés de l'échantillon aléatoires créées en ce qui a trait aux variables sociodémographiques (sexe, âge) et aux résultats aux échelles de psychopathie, de personnalité et d'attitudes quant à la consommation de substances. Les résultats issus de l'analyse factorielle principale utilisant la méthode de rotation promax (compte tenu du lien entre les facteurs primaire et secondaire) appuient partiellement la présence de deux facteurs. Par ailleurs, certains items paraissent problématiques puisqu'ils n'atteignent pas le critère de saturation de 0,30 établi (items 4,10 et 26) ou parce qu'ils s'associent aux deux facteurs théoriques (item 23). Les coefficients de saturation de chaque item sur leur facteur d'appartenance sont présentés au Tableau 2. Le premier facteur explique $17 \%$ de la variance observée dans les résultats au questionnaire et le second, $8 \%$ de la variance. En retirant les items problématiques mentionnés ci-haut $(4,10,23$ et 26$)$ la version en 22 items permet d'obtenir des coefficients alpha de 0,79 pour l'échelle totale, de 0,81 pour l'échelle primaire et de 0,66 pour l'échelle de psychopathie secondaire. Le pourcentage de variance expliqué pour 
le premier facteur s'élève alors à plus de $20 \%$ et à près de $10 \%$ pour le deuxième facteur.

TABLEAU 2

Résultats des analyses factorielles exploratoires et confirmatoires du LSRP

\begin{tabular}{|c|c|c|c|c|c|}
\hline Items & \multicolumn{2}{|c|}{ Facteur primaire } & Items & \multicolumn{2}{c|}{ Facteur secondaire } \\
\hline & AFE & AFC & & AFE & AFC \\
\hline 1 & 0,47 & 0,61 & 17 & 0,33 & 0,49 \\
\hline 2 & 0,46 & 0,60 & 18 & 0,40 & 0,61 \\
\hline 3 & 0,39 & 0,50 & 19 & 0,27 & 0,30 \\
\hline $4^{\text {a }}$ & 0,08 & 0,10 & 20 & 0,24 & 0,31 \\
\hline 5 & 0,43 & 0,53 & 21 & 0,39 & 0,51 \\
\hline 6 & 0,57 & 0,76 & 22 & 0,37 & 0,58 \\
\hline 7 & 0,43 & 0,59 & $23^{\text {a }}$ & 0,16 & 0,18 \\
\hline 8 & 0,46 & 0,63 & 24 & 0,37 & 0,47 \\
\hline 9 & 0,49 & 0,65 & 25 & 0,32 & 0,37 \\
\hline $10^{a}$ & 0,15 & 0,17 & $26^{a}$ & 0,17 & 0,26 \\
\hline 11 & 0,35 & 0,45 & & & \\
\hline 12 & 0,32 & 0,39 & & & \\
\hline 13 & 0,30 & 0,51 & & & \\
\hline 14 & 0,37 & 0,49 & & & \\
\hline 15 & 0,38 & 0,40 & & & \\
\hline 16 & 0,36 & 0,36 & & & \\
\hline
\end{tabular}

$\mathrm{AFE}=$ Analyse factorielle exploratoire $; \mathrm{AFC}=$ analyse factorielle confirmatoire ${ }^{\mathrm{a}}{ }^{\mathrm{a}}=$ item problématique .

Des analyses factorielles confirmatoires sont ensuite effectuées sur la seconde moitié de l'échantillon. Ces analyses visent à vérifier de manière plus rigoureuse la bidimensionalité des composantes de la psychopathie formulée par Levenson et al. (1995). Les modèles sont estimés en utilisant l'option «variables catégorielles» du prologiciel EQS 6.2 (Bentler, 2005; Byrne, 2006). Les résultats obtenus à l'aide de la méthode robuste, en s'appuyant sur le coefficient de Mardia, sont retenus puisqu'ils prennent en compte la non-normalité de la distribution de l'échantillon. Les indices d'ajustement du modèle en deux dimensions corrélées se sont révélés insatisfaisants (CFI $=0,87$ et NNFI $=0,86$, $x^{2} / \mathrm{dl}=5,20$, RMSEA $\left.=0,06\right)$. Par ailleurs, en utilisant la stratégie employée par Lynam et al. (1999), c'est-à-dire en permettant des corré- 
lations entre les termes d'erreur, le modèle obtenu s'avère significatif $\left(\mathrm{CFI}=0,92\right.$, NNFI $=0,91, x^{2} / \mathrm{d} 1=3,71$, RMSEA $\left.=0,05\right)$. Seulement quatre corrélations entre les termes d'erreur ont dû être fixées pour obtenir un bon ajustement (corrélations entre les items 9 et 13, 15 et 16, 19 et 21, 24 et 25) comparativement à 17 dans l'étude de Lynam et al. (1999). Lorsque nous posons les mêmes corrélations entre les termes d'erreur que ce qui a été proposé dans l'étude de Lynam et al. (1999), les coefficients d'ajustement sont légèrement améliorés comparativement au modèle préalablement cité (CFI $=0,94$, NNFI $=0,93$, $x^{2} / d l=3,21$, RMSEA $\left.=0,04\right)$. Les corrélations entre chaque item et son facteur associé sont également rapportées au Tableau 2. Les résultats obtenus corroborent ceux rapportés dans l'analyse factorielle exploratoire. Nous avons également refait les analyses en retirant les items qui s'étaient révélés problématiques selon la théorie classique des tests (items 4, 10, 23 et 26). Les coefficients d'ajustement, en considérant un lien entre les facteurs, mais sans contrainte entre les termes d'erreur, sont insatisfaisants (respectivement CFI $=0,90$, NNFI $=0,89$, $x^{2} / d l=5,44$, RMSEA $\left.=0,06\right)$. Par contre, lorsque les quatre corrélations entre les termes d'erreur mentionnées plus haut sont posées, le modèle à 22 items obtient de bons coefficients d'ajustement (CFI $=0,94$, NNFI $=0,94, x^{2} / d l=3,46$, RMSEA $\left.=0,05\right)$. Le Tableau 3 résume les coefficients d'ajustement obtenus aux différents modèles testés.

TABLEAU 3

Coefficients d'ajustement des différents modèles testés

\begin{tabular}{|l|c|c|c|c|}
\hline Modèles & $\chi^{2} / \mathrm{dl}$ & CFI & NNFI & RMSEA \\
\hline Modèle à 26 items 2 facteurs corrélés & 5,20 & 0,857 & 0,86 & 0,06 \\
\hline $\begin{array}{l}\text { Modèle à 26 items, 2 facteurs corrélés } \\
17 \text { corrélations entre des termes d'erreur }\end{array}$ & 3,71 & 0,92 & 0,91 & 0,05 \\
\hline $\begin{array}{l}\text { Modèle à 26 items, 2 facteurs corrélés, } \\
4 \text { corrélations entre des termes d'erreur }\end{array}$ & 3,21 & 0,94 & 0,93 & 0,04 \\
\hline Modèle à 22 items, 2 facteurs corrélés & 5,44 & 0,90 & 0,89 & 0,06 \\
\hline $\begin{array}{l}\text { Modèle à 22 items, 2 facteurs corrélés } \\
4 \text { corrélations entre les termes d'erreur }\end{array}$ & 3,46 & 0,94 & 0,94 & 0,05 \\
\hline
\end{tabular}

${ }^{1}$ Corrélations entre les termes d'erreur des items 1-5, 1-7, 1-8, 4-5, 5-8, 7-9, 9-13, 10-12, 10-14, 11-13, 12-14, 12-19, 15-16, 19-20, 19-21, 20-23 et 24-25 (Brinkley et al., 2001; Lynam et al., 1999).

${ }^{2}$ Corrélations entre les termes d'erreur des items 15-16, 24-25, 9-13 et 19-21. 


\section{Différence entre les hommes et les femmes}

Pour vérifier l'invariance des modèles chez les hommes et les femmes, une série de tests hiérarchiques est effectuée (Raju, Lafitte \& Byrne, 2002; Schmitt \& Kuljanin, 2008). Le premier test nécessite d'établir un modèle d'invariance configurale afin de déterminer si le nombre de facteurs est identique chez les hommes et les femmes. Le modèle configural vise à comparer la différence entre les coefficients d'ajustement pour le modèle à 26 items, présentant deux facteurs corrélés et quatre corrélations entre les termes d'erreur des hommes et des femmes. Les tests réalisés permettent de comparer les modèles suivants au modèle d'invariance configurale. Les critères utilisés afin de statuer sur l'invariance à chacune des étapes de comparaison des modèles d'invariance, d'homogénéité des variances et d'homogénéité des covariances sont les suivants selon Cheug et Rensvold (2002), Raykov (2004), Chen (2007) et Little, Card, Siegers \& Ledford, (2007): (1) les $\Delta$ CFI ne doivent pas dépasser le 0,01; et (2) le RMSEA doit se situer à l'intérieur de l'intervalle de confiance du modèle de référence. Le premier modèle comparé porte sur l'invariance des saturations de chacun des items pour les modèles des hommes et des femmes (modèle d'invariance du modèle de mesure). À cette étape, bien que les critères précédemment notés soient satisfaits, quatre items présentent des coefficients de saturation différents chez les hommes et les femmes (items 9, 18, 19 et 23). Les contraintes non respectées sont alors retirées afin d'évaluer l'homogénéité des variances des facteurs et des indicateurs en plus des paramètres invariants du modèle d'invariance (modèle d'homogénéité des variances). Enfin, le dernier test consiste à vérifier l'homogénéité des covariances du modèle d'invariance via l'analyse des variances et des covariances entre les facteurs et des paramètres invariants. Le Tableau 4 résume les résultats obtenus aux différentes étapes d'exécution des analyses d'invariance. Dans l'ensemble, les résultats obtenus démontrent que le modèle à 26 items (deux facteurs corrélés, avec quatre corrélations fixées entre les termes d'erreur) est partiellement invariant entre les hommes et les femmes. Le patron de saturation des items 9, 18, 19 et 23 sur leur facteur respectif n'est pas invariant selon le sexe des participants, ce qui signifie qu'ils ne s'associent pas au même facteur selon que le répondant est un homme ou une femme. 
TA B LEA U 4

Coefficients d'ajustement pour les tests hiérarchiques emboîtés d'analyse d'invariance entre les hommes et les femmes

\begin{tabular}{|c|c|c|c|c|c|c|c|c|c|c|c|}
\hline Modèles & CFI & NNFI & RMSEA & S-B $\chi^{2}$ & $\mathrm{dl}$ & $\mathrm{p}$ & IC $90 \%$ & $\Delta \mathrm{S}-\mathrm{B} \chi^{2}$ & $\Delta \mathrm{dl}$ & $\Delta \mathrm{CFI}$ & Contraintes \\
\hline Configural & 0,908 & 0,898 & 0,048 & 2081,37 & 588 & $<0,001$ & {$[0,046,0,050]$} & - & - & - & - \\
\hline $\begin{array}{l}\text { Invariance du } \\
\text { modèle de mesure }\end{array}$ & 0,906 & 0,900 & 0,048 & 2129,73 & 612 & $<0,001$ & {$[0,045,0,050]$} & 48,36 & 24 & 0,002 & $\mathrm{i}_{9}, \mathrm{i}_{19}, \mathrm{i}_{18}, \mathrm{i}_{23}$ \\
\hline $\begin{array}{l}\text { Homogénéité } \\
\text { des variances }\end{array}$ & 0,907 & 0,901 & 0,047 & 2109,44 & 610 & $<0,001$ & {$[0,045,0,050]$} & 28,07 & 22 & 0,001 & - \\
\hline $\begin{array}{l}\text { Homogénéité } \\
\text { des covariances }\end{array}$ & 0,908 & 0,902 & 0,047 & 2108,90 & 615 & $<0,001$ & {$[0,045,0,049]$} & 27,53 & 27 & 0,000 & - \\
\hline
\end{tabular}

RMSEA $=$ Root mean square error of approximation; NNFI $=$ Nonnormed Fit Index; CFI $=$ Comparative Fit Index; S-B $\chi^{2}=$ Satorra \& Bentler scaled chi-square 
La comparaison entre les hommes et les femmes concernant les traits de personnalité psychopathiques totaux, primaires et secondaires avec la version originale du questionnaire à 26 items démontre qu'il existe une différence significative uniquement pour les échelles de psychopathie totale $(F[1,2504]=6,13 ; p<0,05 ; d=0,46)$ et primaire $(F[1,2489]=23,19 ; p<0,001 ; d=0,63)$ entre les hommes et les femmes (Tableau 1).

Les résultats des analyses d'items, des analyses factorielles exploratoires et confirmatoires et des tests d'invariance ont conduit à l'identification de quelques items problématiques. Cependant, ces problèmes métriques n'affectent pas en profondeur les coefficients de fidélité obtenus et la structure interne de l'instrument. Ils sont donc retenus dans le cadre des analyses de validité convergente. Cette décision vise aussi à maximiser la qualité des comparaisons entre la version originale de langue anglaise et la présente adaptation en langue française.

\section{Validité convergente}

Les analyses corrélationnelles effectuées à l'aide de la version originale à 26 items, du NEO-FFI et de l'échelle d'habitudes de consommation de substances sont présentées au Tableau 5. La matrice de corrélations fait état de liens significatifs positifs entre l'échelle totale de psychopathie, le facteur Névrosisme et les habitudes de consommation de substances, et de liens négatifs avec les facteurs Extraversion, Ouverture à l'expérience, Amabilité, et Sens des responsabilités. Plus spécifiquement, l'échelle de psychopathie primaire est associée négativement aux facteurs Amabilité, Ouverture à l'expérience et Sens des responsabilités ainsi que négativement à l'échelle d'habitudes de consommation de substances problématiques. L'échelle de psychopathie secondaire est, quant à elle, reliée positivement au facteur Névrosisme et à l'échelle d'habitudes de consommation de substances problématiques, et négativement aux facteurs Amabilité, Sens des responsabilités et Extraversion. 
TABLEAU 5

Matrice de corrélations entre le LSRP, le NEO-FFI et

les habitudes de consommation

\begin{tabular}{|c|c|c|c|c|c|c|c|c|c|}
\hline Variables & 1 & 2 & 3 & 4 & 5 & 6 & 7 & 8 & 9 \\
\hline 1. Total & 1,00 & & & & & & & & \\
\hline 2. Primaire & $0,87^{* * *}$ & 1,00 & & & & & & & \\
\hline 3. Secondaire & $0,71^{* * *}$ & $0,29 * * *$ & 1,00 & & & & & & \\
\hline 4. Névrosisme & $0,29 * * *$ & 0,04 & $0,54 * * *$ & 1,00 & & & & & \\
\hline 5. Extraversion & $-0,13^{* * *}$ & $-0,03$ & $-0,24^{* * *}$ & $-0,33^{* * *}$ & 1,00 & & & & \\
\hline 6. Ouverture à l'expérience & $-0,17^{* * *}$ & $-0,23^{* * *}$ & 0,01 & 0,08 & 0,03 & 1,00 & & & \\
\hline 7. Amabilité & $-0,60 * * *$ & $-0,49 * * *$ & $-0,46^{* * *}$ & $-0,26^{* * *}$ & $0,23^{* * *}$ & $0,09 * * *$ & 1,00 & & \\
\hline 8. Sens des responsabilités & $-0,37^{* * *}$ & $-0,21^{* * *}$ & $-0,45^{* * *}$ & $-0,25 * * *$ & $0,16^{* * *}$ & $-0,05^{*}$ & $0,32 * * *$ & 1,00 & \\
\hline 9. Habitudes de consommation & $0,29 * * *$ & $0,27^{* * *}$ & $0,20 * * *$ & 0,03 & $0,13^{* * *}$ & 0,02 & $-0,24^{* * *}$ & $-0,24^{* * *}$ & 1,00 \\
\hline
\end{tabular}

${ }^{*} p<0,05 ;{ }^{* *} p<0,01 ;{ }^{* * *} p<0,001$ 


\section{Discussion}

L'objectif de cette étude était de réaliser une démarche de validation de la version française du LSRP auprès d'une clientèle de jeunes adultes en évaluant: (a) le degré d'association de chacun des items à leur facteur latent; (b) la structure factorielle de l'instrument; (c) la différence entre les hommes et les femmes; et (d) la validité de convergence de l'instrument. Les résultats appuient généralement bien la structure en deux facteurs de l'instrument en version française. En effet, les résultats des analyses factorielles exploratoires sont comparables à ce qui a été obtenu par les auteurs de l'instrument et dans d'autres études (Levenson et al., 1995; Lynam et al., 1999). De plus, la plupart des items sont correctement associés à leur facteur latent, hormis les items 4, 10, 23 et 26 qui ne satisfont pas aux critères de «bons items» selon la théorie classique des tests et qui ne saturent pas de façon significative sur leur facteur latent. Les items 10 et 23 sont des items à sens inversé, ce qui pourrait expliquer les indices plus faibles obtenus. En effet, bon nombre d'auteurs ont documenté que de tels items peuvent semer la confusion chez les répondants et donc offrir des réponses moins consistantes. Ceci se répercute inévitablement tant sur la structure factorielle que sur la cohérence interne d'un instrument (Swain, Weathers \& Niedrich, 2008). L'item 4, «Mon but principal dans la vie, c'est d'aller chercher le plus de bonnes choses possible» pourrait très bien ne pas être un item discriminant de traits psychopathiques, mais mesurer davantage le positivisme. Pour l'item 26, «On surestime l'amour», il se pourrait qu'il ne soit pas conceptuellement relié au construit, ce qui remet en question sa présence dans l'échelle.

Les résultats qui émergent des analyses factorielles confirmatoires laissent également présager que le modèle en deux facteurs est optimal auprès d'une population générale. La nécessité de fixer des corrélations entre les termes d'erreur afin d'obtenir de bons indices d'ajustement demeure néanmoins un désavantage du modèle, bien que le nombre fixé dans la présente étude soit considérablement inférieur à ce qui a été obtenu avec la version américaine du LSRP (Lynam et al., 1999). Cela signifie que l'on accepte que deux items possèdent des éléments qui corrèlent ensemble, mais qui ne sont pas en lien avec le construit mesuré. Une telle procédure peut néanmoins se justifier lorsque la corrélation est théoriquement explicable, ce qui est d'ailleurs le cas dans la présente étude (Bagozzi, 1983; Fornell, 1983; Reddy, 1992). En 
effet, les items 9 et 13 partagent des aspects communs d'une certaine forme de sadisme, un autre construit souvent lié à la psychopathie. Nous ne pouvons non plus exclure la possibilité que ces items soient plus sensibles à la désirabilité sociale et cela pourrait expliquer les corrélations nécessaires entre les termes d'erreur. La corrélation entre les items 15 et 16 pourrait s'expliquer par l'association à un autre construit, soit la bienveillance ou l'honnêteté envers les gens. Toutefois, l'hypothèse explicative la plus probable est que les deux items sont à sens inversé et se ressemblent beaucoup, ce qui a pu donner lieu à des styles d'endossement similaires. Les items 19 et 21 sous-tendent une notion de persistance et d'effort en plus de mesurer l'impulsivité. Enfin, les items 24 et 25 évaluent tous les deux la gestion de la colère. Des problèmes de saturation entre les items précédemment nommés et leur facteur latent pourraient également expliquer en partie les coefficients d'ajustement et de cohérence interne plus faibles obtenus.

Pour ce qui est de la différence hommes-femmes, les modèles mesurés semblent partiellement invariants selon le genre des participants. Nous notons, par exemple, que les coefficients de saturation des items 9 , 18, 19 et 23 sont différents selon le sexe. Après avoir reproduit les analyses factorielles exploratoires séparément pour les hommes et les femmes, chez les hommes, l'item 9 obtient des coefficients de saturation équivalents sur les deux facteurs alors que les items 18 et 19 ne s'associent pas au bon facteur théorique, ce qui n'est pas le cas chez les femmes. Pour ce qui est de l'item 23, il est associé au facteur $1 \mathrm{chez}$ les hommes alors que pour les femmes, il est associé aux deux facteurs de façon équivalente. Il semble donc que ces items aient une connotation différente pour les hommes et les femmes. L'item 9, «Je dis aux autres ce qu'ils veulent bien entendre pour les amener à faire ce que je veux", semble un signe de manipulation chez les femmes, alors que chez les hommes, il semble autant relié à l'aspect comportemental (secondaire) qu'aux attitudes associées au construit de psychopathie (primaire). Les items 18 et 19, «J'éprouve souvent de l'ennui» et «Je me sens capable de poursuivre un même but sur une longue période de temps», ne semblent pas des indicateurs comportementaux efficaces pour identifier cette composante du construit de psychopathie chez les hommes. Enfin, l'item 23, «Avant de faire quoi que ce soit, j'en pèse toutes les conséquences possibles», semble davantage être considéré comme étant un indicateur de planification sournoise pour parvenir à ses fins par les hommes que comme une incapacité à contenir l'impulsivité, alors que 
les femmes répondent de façon à ce que cet item puisse représenter les aspects tant manipulatoire que comportemental de la psychopathie. Une compréhension différente des items entre les hommes et les femmes a également été observée dans des études sur le PCL-R (Harpur et al., 1989, Salekin et al., 1997). Les auteurs ont alors souligné qu'un modèle à trois facteurs s'appliquerait davantage aux données recueillies auprès des femmes que le modèle original à deux facteurs (Salekin, Rogers \& Sewell, 1997; Warren et al., 2003). Des analyses utilisant la théorie de réponse aux items suggèrent également une différence quant au fonctionnement différentiel des items chez les hommes et les femmes, sans toutefois que cet écart soit suffisant pour avoir un impact majeur sur le résultat total au questionnaire (Bolt, Hare, Vitale \& Newman, 2004). Enfin, il a également été envisagé que les patrons de réponse aux questionnaires évaluant les traits psychopathiques soient différents surtout entre les hommes et les femmes provenant d'une population clinique (Brinkley et al., 2008). À cet égard, d'autres recherches nécessitent d'être menées afin de mieux comprendre comment cette distinction hommes-femmes s'actualise dans le mode de réponse au LSRP.

Les hommes de l'échantillon présentent des résultats plus élevés aux échelles de psychopathie totale et primaire que les femmes, ce qui appuie la position de plusieurs auteurs sur le sujet, à savoir que les hommes sont plus enclins à user de manipulation, de tromperie, de charme superficiel et à être insensibles que les femmes. Il s'agit là en effet d'une distinction conceptuelle couramment mentionnée dans la documentation scientifique. Néanmoins, certains auteurs remettent en question le postulat implicite selon lequel le concept de psychopathie, plus souvent étudié chez les hommes, se transpose parfaitement chez les femmes (Forouzan \& Cooke, 2005; Jackson \& Richards, 2007; Verona \& Vitale, 2006). Ils notent plusieurs différences possibles chez les femmes: la manipulation se ferait davantage par une attitude séductrice plutôt que par l'arnaque; la grandiosité et le charme superficiel seraient observés uniquement dans les cas extrêmes; la promiscuité sexuelle servirait à des fins d'exploitation de l'autre plutôt que pour rechercher des sensations fortes (facteur 2; Quinsey, 2002).

Par contre, aucune différence significative n'est décelée pour ce qui est de l'échelle de psychopathie secondaire dans notre échantillon. C'est donc dire que l'expression comportementale des traits psychopathiques chez les jeunes adultes s'observe de la même façon chez les hommes et les femmes, c'est-à-dire par une propension à être impulsif, à répéter le 
même genre d'erreurs d'une fois à l'autre et à présenter des comportements antisociaux.

Enfin, les résultats issus de l'analyse de validité du LSRP appuient la position selon laquelle les traits primaires sont principalement associés à un faible degré d'amabilité, auquel on ajoute une faible ouverture à l'expérience et peu de préoccupations pour les responsabilités. Pour ce qui est des traits de psychopathie secondaire, ils correspondent également à un faible degré d'amabilité et de sens des responsabilités, mais aussi à un manque d'extraversion et surtout, à la forte présence d'instabilité émotionnelle (Névrosisme). Ces résultats soutiennent partiellement l'approche selon laquelle les gens présentant des traits de psychopathie primaire obtiendraient des résultats faibles au facteur Amabilité et ceux possédant des traits de psychopathie secondaire auraient des résultats faibles aux facteurs Amabilité et Sens des responsabilités (Lynam et al., 1999; Miller, Lynam, Widiger \& Leukefeld, 2001; Snyder \& Regts, 1990; Widiger \& Lynam, 2003). Le facteur Névrosisme est quant à lui parfois cité comme étant également caractéristique des individus présentant des traits de psychopathie, plus particulièrement de psychopathie secondaire (Hart \& Hare, 1994; Lykken, 1995; Lynam et al., 1999; Miller et al., 2001 ; Patrick, 1994; Snyder \& Regts, 1990). Il semble par ailleurs que les habitudes de consommation de substances problématiques soient davantage en lien avec la psychopathie primaire qu'avec la psychopathie secondaire, ce qui est étonnant compte tenu du caractère comportemental de la seconde échelle. Par contre, des résultats similaires avaient été obtenus par Lynam et al. (1999). Lorsque les analyses corrélationnelles sont effectuées séparément pour les hommes et les femmes, il semble que la consommation soit davantage liée à la psychopathie primaire chez les hommes, mais qu'elle soit autant reliée à la psychopathie primaire que secondaire chez les femmes (respectivement $r=0,18$; $p \leq 0,01$ et $r$ $=0,20 ; p \leq 0,01)$. Ce résultat contre-intuitif pourrait être attribuable au fait que notre échantillon est constitué d'adolescents et de jeunes adultes. Certains participants présentant des traits psychopathiques primaires en sont peut-être à leurs premières expériences de consommation de substances, étape qui sera brève pour eux alors qu'elle pourrait se chroniciser chez les participants affichant des traits psychopathiques secondaires, donnant ainsi lieu au constat observé chez les populations adultes (Lynam et al., 1999). 
Malgré ses aspects prometteurs, la présente étude comporte des limites. Par exemple, la représentativité de l'échantillon n'est pas assurée. En effet, il s'agissait d'un échantillon de jeunes adultes chez qui les traits psychopathiques pourraient être plus importants compte tenu de l'étape développementale dans laquelle ils se trouvent et du degré d'impulsivité y étant associé. Ainsi, il serait important de suivre les adolescents et les jeunes adultes sur une longue période de temps afin d'étayer les données quant au pouvoir prédictif de l'instrument. De plus, il serait essentiel de répliquer les analyses discutées dans le présent article auprès de clientèles plus diversifiées. Deuxièmement, la mesure de consommation de substances utilisée dans la présente étude comme variable pour évaluer la validité convergente du LSRP n'est pas un instrument standardisé. D’autres instruments mesurant spécifiquement ce construit devraient être inclus dans de futures recherches pour appuyer la validité convergente. Enfin, l'étude actuelle ne s'attaque pas directement à l'épineux problème de la désirabilité sociale. Bien que le questionnaire ait été construit afin de minimiser ce biais de réponse, la transparence de certains items pourrait néanmoins influencer la façon de répondre aux questions. Ainsi, d'autres études prenant en compte la désirabilité sociale et les traits psychopathiques mesurés par le LSRP devraient être effectuées.

Somme toute, l'échelle auto-rapportée de psychopathie présente les mêmes caractéristiques que celles de la version originale anglaise, allant même jusqu'à partager les mêmes problèmes (Brinkley et al., 2001; Sellbom, 2011). Dans cette perspective, des études plus fines de la structure factorielle de l'instrument et une évaluation encore plus spécifique des items représentent des avenues de recherche incontournables. De plus, notre étude a permis d'identifier les mêmes items problématiques que dans la version originale anglaise, c'est-à-dire ceux qui ne permettent pas de bien discriminer entre les individus et qui ne corrèlent pas de façon acceptable à leur facteur latent (Brinkley et al., 2008; Sellbom, 2011). Or, ces conclusions sont issues uniquement d'analyses factorielles. Des analyses d'items plus approfondies, utilisant notamment la théorie de réponse aux items, semblent une avenue de recherche intéressante afin de mieux documenter les courbes caractéristiques d'items et les courbes caractéristiques d'options. Dans un tel cas, une révision des items considérés comme problématiques devrait être envisagée, accompagnée d'une nouvelle validation de l'instrument. 
Malgré ses limites conceptuelles, le LRSP demeure un bon instrument de dépistage des traits psychopathiques. À l'heure actuelle, il s'agit d'une mesure auto-rapportée brève, en langue française, qui préserve les propriétés métriques de la version américaine originale. Le questionnaire constitue donc une option intéressante pour appuyer l'évaluation et le dépistage des traits psychopathiques de la personnalité ainsi que des problèmes de comportement reliés à la délinquance, la criminalité, l'impulsivité et la violence y étant associés. Il existe d'autres instruments fondés sur le rapport verbal des participants qui sont fort valables, mais ceux-ci sont beaucoup plus longs (p. ex. Psychopatbic Personality Inventory; Lilienfeld \& Andrews, 1996) ou n'ont pas été validés en langue française (p. ex. le Dirty Dozen; Jonason \& Webster, 2010; ou encore le Self-Reported Psychopathy Scale-II; Hare, Harpur, \& Hemphill, 1989). Pour le reste, il s'agit d'instruments dont l'administration nécessite une procédure de cotation par un professionnel qui ne s'appliquent pas nécessairement à tous les contextes, dont en recherche (p. ex. Psychopatby Checklist-Revised; Hare, 1991, 2003). Les professionnels pourraient, entre autres, utiliser le LSRP afin de détecter des traits de personnalité pathologiques et de les approfondir après coup à l'aide de mesures cliniques plus élaborées. Il constitue également un bon outil en recherche, lorsque la concision est requise. L'instrument pourrait profiter de certaines améliorations en ce qui a trait à la composition et à la sélection des items. Par exemple, les quatre items problématiques relevés pourraient être éventuellement reformulés ou retirés de l'échelle. D'autres études sur la généralisation de nos résultats devront être menées avant de déterminer la valeur de ces solutions de rechange.

\section{Références}

Babiak, P., \& Hare, R. D. (2007). Snakes in suits: When psychopaths go to work. New York, NY: HaperCollins Publishers Inc.

Bagozzi, R. P. (1983). Issues in the Application of Covariance Structure Analysis: A Further Comment. Journal of Consumer Research, 9, 449-450. doi: $10.1086 / 208939$

Benning, S. D., Patrick, C. J., Hicks, B. M., Blonigen, D. M., \& Krueger, R. F. (2003). Factor structure of the Psychopathic Personality Inventory: Validity and implications for clinical assessment. Psychological Assessment, 15, 340-350. doi : $10.1037 / 1040-3590.15 .3 .340$

Bentler, P. M. (2005). EQS structural equation program model. Enrico, CA: Multivariate Software. 
Blonigen, D. M., Carlson, M. D., Hicks, B. M., Krueger, R. F., \& Iacono, W. G. (2008). Stability and change in personality traits from late adolescence to early adulthood: A longitudinal twin study. Journal of Personality, 76, 229266. doi: $10.1111 /$ j.1467-6494.2007.00485.x

Bolt, D., Hare, R. D., Vitale, J. E., \& Newman, J. P. (2004). A multigroup item response theory analysis of the Psychopathy Checklist-Revised. Psychological Assessment, 16, 155-168. doi : 10.1037/1040-3590.16.2.155

Brinkley, C. A., Newman, J. P., Widiger, T. A., \& Lynam, D. R. (2004). Two approaches to parsing the heterogeneity of psychopathy. Clinical Psychology: Science and Practice, 11, 69-94. doi : 10.1093/clipsy/bph054

Brinkley, C. A., Diamon, P. M., Magaletta, P. R., \& Heigel, C. P. (2008). CrossValidation of Levenson's Psychopathy Scale in a sample of federal female inmates. Assessment, 15, 464-482. doi : 10.1177/1073191108319043

Brinkley, C. A., Schmitt, W. A., Smith, S. S., \& Newman, J. P. (2001). Construct validation of a self-reported psychopathy scale: Does Levenson's selfreported psychopathy scale measure the same constructs as Hare's psychopathy checklist-revised? Personality and Individual Differences, 31, 1021-1038. doi : 10.1016/S0191-8869(00)00178-1

Buss, D. (1993). Ways to curtail employee theft. Nation's Business, 81, 36-37.

Byrne, B. M. (1994). Structural equation modeling with EQS and EQS/Windows: Basic concepts, applications, and programming. Thousand Oaks, CA: Sage.

Byrne, B. M. (2006). Structural equation modeling with EQS and EQS/Windows: Basic concepts, applications, and programming (2 ${ }^{\text {ème }}$ éd.). Thousand Oaks, CA: Sage.

Caponecchia, C., Sun, A.Y.Z., \& Wyatt, A. (2012). "Psychopaths" at work? Implications of lay persons' use of labels and behavioural criteria for psychopathy. Journal of Business and Ethic, 107, 399-408. doi : 10.1007/s10551011-1049-9

Chen, F. F. (2007). Sensitivity of goodness of fit indexes to lack of measurement invariance. Structural Equation Modeling, 14, 464-504. doi: 10.1080/ 10705510701301834

Cheung, G. W., \& Rensvold, R. B. (2002). Evaluating goodness-of-fit indexes for testing measurement invariance. Structural Equation Modeling, 9, 233-255. doi : 10.1207/S15328007SEM0902_5.

Cleckley, H. (1976). The mask of sanity (5 $5^{\text {th }}$ ed.). St. Louis, MO: Mosby.

Costa, P. T., \& McCrae, R. R. (1992). Revised NEO Personality Inventory (NEO$P I-R)$ and Five-Factor Inventory (NEO-FFI) professional manual. Odessa, FL: Psychological Assessment Resources.

Costa, P. T., \& McCrae, R. R. (2010). NEO PI-3: Professional manual: Revised NEO PI-3 and NEO-FFI-3. Florida: Psychological Assessment Resources, Inc.

Decuyper, M., De Clercq, B., De Bolle, M., \& De Fruyt, F. (2009). Validation of FFM PD counts for Screening personality pathology and psychopathy in adolescence. Journal of Personality Disorders, 23, 587-605. doi: 10.1521/ pedi.2009.23.6.587 
Egan, V. \& Angus, S. (2004). Is social dominance a sex-specific strategy for infidelity? Personality and Individual Differences, 36, 575-586. doi : 10.1016/ S0191-8869(03)00116-8

Fornell, C. (1983). Issues in the Application of Covariance Structure Analysis: A Comment. Journal of Consumer Research, 9, 443-447. doi : 10.1086/208938

Forouzan, E., \& Cooke, D. J. (2005). Figuring out la femme fatale: Conceptual and assessment issues concerning psychopathy in females. Behavioral Sciences and the Law, 23, 765-778. Doi : 10.1002/bsl.669.

Forth, A. E., Brown, S. L., Hart, S. D., \& Hare, R. D. (1996). The assessment of psychopathy in male and female noncriminals: Reliability and validity. Personality and Individual Differences, 20, 531-543. doi: 10.1016/01918869(95)00221-9

Gummelt, H. D., Anestis, J. C., \& Carbonell, J. L. (2012). Examining the Levenson Self Report Psychopathy Scale using a Graded Response Model. Personality and Individual Differences, 53, 1002-1006. doi: 10.1016/j. paid.2012.07.014

Hall, J. R. \& Benning, S. D. (2006). The "Successful” Psychopath: Adaptive and Subclinical Manifestations of Psychopathy in the General Population. Dans C. J. Patrick (Éd.), Handbook of psychopathy (pp. 459-478). New York, NY: The Guilford Press.

Hare, R. D. (1991). The Hare Psychopathy Checklist-Revised (PCL-R). Toronto: Multi-Health Systems.

Hare, R. D. (1993). Without conscience: The disturbing world of the psychopaths among us. New York, NY: The Guilford Press.

Hare, R. D. (2003). The Hare Psychopathy Checklist-Revised. Toronto: MultiHearlth Systems.

Hare, R. D., Harpur, T. J., \& Hemphill, J. D. (1989). Scoring pamphlet for the Self-Report Psychopatby scale: SRP-II. (Document inédit). Vancouver: Simon Fraser University.

Harpur, T. J., Hare, R. D., \& Hakistian, A. R. (1989). Two-factor conceptualization of psychopathy: Construct validity and assessment implications. Psychological Assessment, 1, 6-17.

Harpur, T. J., Hakstian, A. R., \& Hare, R. D. (1988). Factor structure of the Psychopathy Checklist. Journal of Consulting and Clinical Psychology, 56, 741747. doi : 10.1037/0022-006X.56.5.741

Hart, S. D. \& Hare, R. D. (1994). Psychopathy and de big 5: Correlations beween observers' ratings of normal and pathological personality. Journal of Personality Disorders, 8, 32-40. doi : 10.1521/pedi.1994.8.1.32

Hill, C. D., Neumann, C. S., \& Rogers, R. (2004). Confirmatory factor analysis of the Psychopathy Checklist: Screening version in offenders with Axis I disorders. Psychological Assessment, 16, 90-95. doi: 10.1037/10403590.16.1.90.

Holtzworth-Munroe, A., Meehan, J. C., Herron, K., Rehaman, J. C., \& Stuart, G. L. (2003). Do subtypes of maritally violent men continue to differ over 
time? Journal of Consulting and Clinical Psychology, 71, 728-740. doi: 10.1037/0022-006X.71.4.728

$\mathrm{Hu}, \mathrm{L} .$, \& Bentler, P. M. (1999). Cutoff criteria for fit indexes in covariance structure analysis: Conventional criteria versus new alternatives. Structural Equation Modeling, 6, 1-55. doi: 10.1080/10705519909540118

Jackson, R., \& Richards, H. (2007). Psychopathy in women: A valid construct with clear implications. Dans H. Hervé \& J. C. Yuille (Éds.), The psychopath: Theory, research, and practice (pp. 389-410). Mahwah, NJ: Lawrence Erlbaum Associates Publishers.

Jonason, P. K. \& Buss, D. M. (2012). Avoiding entangling commitments: Tactics for implementing a short-term mating strategy. Personality and Individual Differences, 52, 606-610. doi: 10.1016/j.paid.2011.12.015

Jonason, P. K., \& Webster, G. D. (2010). The Dirty Dozen: A Concise measure of the Dark Triad. Psychological Assessment, 22, 420-432. doi: 10.1037/ a0019265

Karpman, B. (1948). The myth of the psychopathic personality. American Journal of Psychiatry, 104, 523-534.

Kastner, R. M., \& Sellbom, M. (2012). Hypersexuality in college strudents: The role of psychopathy. Personality and Individual Differences, 53, 644-649. doi : $10.1016 /$ j.paid.2012.05.005

Kline, R. B. (2010). Promise and pitfalls of structural equation modeling in gifted research. Dans B. Thompson \& R. F. Subotnik (Éds), Methodologies for conducting research on giftedness (pp. 147-169). Washington, DC: American Psychological Association.

Lee, K., \& Ashton, M. C. (2005). Psychopathy, machiavellianism, and narcissism in the five-factor model and the HEXACO model of personality structure. Personality and Individual Differences, 38, 1571-1582. doi : 10.1016/j. paid.2004.09.016

Lee, Z. \& Salekin, R. T. (2010). Personality Disorders: Theory, Research, and Treatment, 1, 153-169. doi : 10.1037/a0019269

Levenson, M. R., Kiehl, K. A., \& Fitzpatrick, C. M. (1995). Assessing psychopathic attributes in a noninstitutionalized population. Journal of Personality and Social Psychology, 68, 151-158. doi : 10.1037/0022-3514.68.1.151

Lilienfeld, S. O., \& Andrews, B. P. (1996). Development and preliminary validation of a self-report measure of psychopathic personality traits in noncriminal populations. Journal of Personality Assessment, 66, 488-524. doi: 10.1207/s15327752jpa6603_3

Little, T. D., Card, N. A., Slegers, D. W., \& Ledford, E. C. (2007). Modeling contextual effects with multiple-group MACS models. Dans T. D. Little, J. A. Bovaird, \& N. A. Card (Éds.), Modeling ecological and contextual effects in longitudinal studies of buman development (pp. 121-147). Mahwah, NJ: Erlbaum.

Lykken, D. T. (1995). The Antisocial Personalities. Hillsdale, NJ: Erlbaum.

Lynam, D. R., Caspi, A., Moffitt, T. E., Raine, A., Loeber, R. \& StouthamerLoeber, M. (2005). Adolescent Psychopathy and the Big Five: Results from 
Two Samples. Journal of Abnormal Child Psychology, 33, 431-443. doi: 10.1007/s10648-005-5724-0

Lynam, D. R., Whiteside, S. \& Jones, S. (1999). Self-reported psychopathy: A validation study. Journal of Personality Assessment, 73, 110-132. doi : 10.1207/ S15327752JPA730108

Marion, B. E. \& Sellbom, M. (2012). An examination of Gender-Moderated test bias on the Levenson Self-Report Psychopathy Scale. Journal of Personality Assessment, 93, 235-243. doi : 10.1080/00223891.2011.558873

Miller, J. D., Gaughan, E. T., \& Pryor, L. R. (2008). The Levenson Self-Report Psychopathy Scale: An examination of the personality traits and disorders associated with the LSRP factors. Assessment, 15, 450-463. doi: 10.1177/ 1073191108316888

Miller, J. D., Lynam, D. R., Widiger, T. A., \& Leukefeld, C. (2001). Personality disorders as extreme variants of common personality dimensions: Can the Five-Factor Model adequately represent psychopathy? Journal of Personality, 69, 253-276. doi: 10.1111/1467-6494.00144

O'Boyle, E. H., Forsyth, D. R., Banks, G. C., \& McDaniel, M. A. (2012). A meta-analysis of the dark triad and work behavior: A social exchange perspective. Journal of Applied Psychology, 97, 557-579. doi: 10.1037/ a0025679

Patrick, C. J. (1994). Emotion and psychopathy: Starling new insights. Psychophysiology, 31, 415-428. doi : 10.1111/j.1469-8986.1994.tb02440.x

Paulhus, D. L., \& Williams, K. M. (2002). The dark triad of personality: Narcissism, machiavellianism, and psychopathy. Journal of Research in Personality, 36, 556-563. doi : 10.1016/S0092-6566(02)00505-6

Poon, J. M. L. (2003). Situational antecedents and outcomes of organizational politics perceptions. Journal of Managerial Psychology, 18, 138-155. doi: 10.1108/02683940310465036

Quinsey, V. L. (2002). Evolutionary theory and criminal behaviour. Legal and Criminological Psychology, 7, 1-13. doi: 10.1348/135532502168324

Raju, N. S., Laffitte, L. J., \& Byrne, B. M. (2002). Measurement equivalence: A comparison of methods based on confirmatory factor analysis and item response theory. Journal of Applied Psychology, 87, 517-529. doi : 10.1037/00219010.87.3.517

Raykov, T. (2004). Behavioral scale reliability and measurement invariance evaluation using latent variable modeling. Behavior Therapy, 35, 299-331. doi : 10.1016/S0005-7894(04)80041-8

Reddy, S. K. (1992). Effects of ignoring correlated measurement error in structural equation models. Education and Psychological Measurment, 52, 449570. doi : $10.1177 / 0013164492052003005$

Ribeiro da Silva, D., Rijo, D., \& Salekin, R. T. (2013). Child and adolescent psychopathy: Assessment issues and treatment needs. Aggression and violent behavior, 18, 71-78. doi: 10.1016/j.avb.2012.10.003

Sabourin, S., \& Lussier, Y. (1992). French translation of the Five-Factor Inventory (NEO-FFI). (Document inédit).Québec: Université Laval. 
Sabourin, S., \& Lussier, Y. (1998). Traduction du Levenson Self-Reported Psychopathy Scale. (Document inédit). Québec: Université Laval.

Salekin, R. T., \& Lynam, D. R. (2010). Child and adolescent psychopathy: An introduction. Dans R. T. Salekin \& D. R. Lynam (Éds.), Handbook of child and adolescent psychopathy (pp. 1-12). New York, NY: Guilford Press.

Salekin, R. T., Rogers, R., \& Sewell, K. W. (1997). Construct validity of psychopathy in female offender sample: A multitrait-multimethod evaluation. Journal of Abnormal Psychology, 106, 576-585. doi: 10.1037/0021843X.106.4.576

Salekin, R. T., Rosenbaum, J. \& Lee, Z. (2012). Child and Adolescent Psychopathy: Stability and Change. Psychiatry, Psychology and Law, 15, 224-236. doi : $10.1080 / 13218710802014519$

Salekin, R. T., Trobst, K. K., \& Krioukova, M. (2001). Construct validity of psychopathy in a community sample: A nomological net approach. Journal of Personality Disorders, 15, 425-441. doi: 10.1521/pedi.15.5.425.19196

Saunders, J. B., Aasland, O. G., Babor, T. F., de la Fuente, J. R., \& Grant, M. (1993). Development of the Alcohol Use Disorders Identification Test (AUDIT): WHO Collaborative Project on Early Detection of Persons with Harmful Alcohol Consumption-II. Addiction, 88, 791-804. doi: 10.1111/ j.1360-0443.1993.tb02037.x

Savard, C., Lussier, Y., Sabourin, S., \& Brassard, A. (2005, juin). French Canadian validation of the Levenson self-report psychopatby scale. Affiche présentée au $66^{\text {ème }}$ congrès annuel de la Société canadienne de psychologie. Montréal.

Savard, C., Sabourin, S., \& Lussier, Y. (2004, novembre). La relation entre les traits de personnalité psychopathiques et la détresse conjugale. Affiche présentée à la rencontre scientifique annuelle du Centre de recherche interdisciplinaire sur les problèmes conjugaux et les agressions sexuelles (CRIPCAS). Montréal.

Savard, C., Sabourin, S., \& Lussier, Y. (2006). Male sub-threshold psychopathic traits and couple distress. Personality and Individual Differences, 40, 931-942. doi : $10.1016 /$ j.paid.2005.10.001

Savard, C., Sabourin, S., \& Lussier, Y. (2011). Correlates of psychopathic traits in community couples. Personality and Mental Health, 5, 186-199. doi: 10.1002/pmh.159

Schmitt, N., \& Kuljanin, G. (2008). Measurement invariance: Review of practice and implications. Human Resource Management Review, 18, 210-222. doi : 10.1016/j.hrmr.2008.03.003

Sellbom, M. (2011). Elaborating on the construct validity of the Levenson Self-Reported Psychopathy Scale in incarcerated and non-incarcerated samples. Law and Human Behavior, 35, 440-451. doi : 10.1007/s10979-0109249-X

Snyder, D. K. \& Regts, J. M. (1990). Personality correlates of marital dissatisfaction: A comparison of psychiatric, maritally distressed, and nonclinic samples. Journal of Sex and Marital Therapy, 16, 34-43. doi: 10.1080/ 00926239008405964 
Stevens, G. W., Deuling, J. K., \& Armenakis, A. A. (2012). Successful Psychopaths: Are They Unethical Decision-Makers and Why?. Journal of Business Ethics, 105, 139-149, doi : 10.1007/s10551-011-0963-1»10.1007/s10551011-0963-1

Strand, S., \& Belfrage, H. (2005). Gender differences in psychopathy in a Swedish offender sample. Behavioral Sciences $\mathcal{F}$ the Law, 23, 837-850. doi: $10.1002 /$ bsl.674

Swain, S. D., Weathers, D., \& Niedrich, R. W. (2008). Assessing three sources of misresponse to reversed Likert items. Journal of Marketing Research, 45, 116-131. doi : 10.1509/jmkr.45.1.116

Tabachnik, B. G., \& Fidell, L. S. (2001). Using multivariate statistics (4 ${ }^{\text {ème }}$ éd). Needham Heights, MA: Allyn \& Bacon.

Tepper, J. B. (2007). Abusive supervision in work organizations: Review, synthesis, and research agenda. Journal of Management, 33, 261-294. doi: $10.1177 / 0149206307300812$

Tomarken, A. J., \& Waller, N. G. (2003). Potential problems with "well fitting" models. Journal of Abnormal Psychology, 112, 578-598. doi : 10.1037/0021843X.112.4.578

Vachon, D. D., Lynam, D. R., Widiger, T. A., Miller, J. D., McCrae, R. R., \& Costa, P. T. (2013). Basic traits predict the prevalence of personality disorder across the life span: The example of psychopathy. Psychological Science, 24, 698-705. doi: 10.1177/0956797612460249

Verona, E., \& Vitale, J. (2006). Psychopathy in women: Assessment, manifestations, and etiology. Dans C. J. Patrick (Éd.), Handbook of psychopathy (pp. 415-436). New York, NY: Guilford.

Warren, J. I., Burnette, M. L., South, S. C., Chauhan, P., Bale, R., Friend, R., \& Van Patten, I. (2003). Psychopathy in women: Structural modeling and comorbidity. International Journal of Law and Psychiatry, 26, 223-242. doi: 10.1016/S0160-2527(03)00034-7

Widiger, T. A. (1998). Psychopathy and normal personality. Dans D. J. Cooke, A. E. Forthe, \& R. D. Hare (Éds.), Psychopathy: Theory, research and implications for society (pp. 47-68). Dordrecht: Kluwer Academic Publishing.

Widiger, T. A., \& Lynam, D. R. (2003). Psychopathy and the Five-Factor Model of personality. Dans T. Millon, E. Simonsen, M. Birket-Smith, \& R. D. Davis (Éds.), Psychopatby: Antsocial, criminal, and violent behaviour (pp. 171-187). New York, NY: The Guilford Press. 


\section{Annexe A}

\section{L'Échelle auto-rapportée de psychopathie de Levenson et les données descriptives obtenues par les hommes et les femmes à chacun des items de l'instrument.}

\begin{tabular}{|c|c|c|c|c|c|}
\hline & Items & Hommes & Femmes & Total & $F$ \\
\hline 1. & $\begin{array}{l}\text { Le succès est fondé sur la loi du } \\
\text { plus fort; je ne me soucie pas } \\
\text { des perdants. }\end{array}$ & $\begin{array}{l}1,96 \\
(0,94)\end{array}$ & $\begin{array}{l}1,53 \\
(0,75)\end{array}$ & $\begin{array}{l}1,68 \\
(0,84)\end{array}$ & $41,37^{* * *}$ \\
\hline 2. & $\begin{array}{l}\text { Pour moi, tout est correct du } \\
\text { moment que je m'en tire bien. }\end{array}$ & $\begin{array}{c}2,32 \\
(0,95) \\
\end{array}$ & $\begin{array}{c}2,11 \\
(0,88) \\
\end{array}$ & $\begin{array}{c}2,17 \\
(0,90) \\
\end{array}$ & $30,83^{* * *}$ \\
\hline 3. & $\begin{array}{l}\text { Dans le monde d'aujourd'hui, } \\
\text { je me sens justifié(e) de faire } \\
\text { n'importe quoi pour réussir. }\end{array}$ & $\begin{array}{c}2,18 \\
(0,94)\end{array}$ & $\begin{array}{c}1,96 \\
(0,95)\end{array}$ & $\begin{array}{c}2,04 \\
(0,95)\end{array}$ & $0,84^{* * *}$ \\
\hline 4. & $\begin{array}{l}\text { Mon but principal dans la vie, } \\
\text { c'est d'aller chercher le plus de } \\
\text { bonnes choses possible. }\end{array}$ & $\begin{array}{c}3,42 \\
(0,79)\end{array}$ & $\begin{array}{c}3,54 \\
(0,73)\end{array}$ & $3,5(0,75)$ & $9,65^{* * *}$ \\
\hline 5. & $\begin{array}{l}\text { Mon premier but, c'est de faire } \\
\text { beaucoup d'argent. }\end{array}$ & $\begin{array}{c}2,53 \\
(0,88)\end{array}$ & $\begin{array}{c}2,29 \\
(0,85)\end{array}$ & $\begin{array}{c}2,37 \\
(0,87)\end{array}$ & $2,74^{* * *}$ \\
\hline 6. & $\begin{array}{l}\text { Je laisse aux autres le souci des } \\
\text { belles valeurs; moi, je me } \\
\text { préoccupe du résultat final. }\end{array}$ & $\begin{array}{l}1,88 \\
(0,85)\end{array}$ & $\begin{array}{l}1,49 \\
(0,70)\end{array}$ & $\begin{array}{c}1,62 \\
(0,77)\end{array}$ & $12,82^{* * *}$ \\
\hline 7. & $\begin{array}{l}\text { En général, les gens qui sont } \\
\text { assez stupides pour se faire } \\
\text { avoir le méritent. }\end{array}$ & $\begin{array}{l}2,00 \\
(0,93)\end{array}$ & $\begin{array}{l}1,63 \\
(0,80)\end{array}$ & $\begin{array}{l}1,76 \\
(0,87)\end{array}$ & $3,24^{* * *}$ \\
\hline 8. & $\begin{array}{l}\text { Ma priorité absolue, ce sont mes } \\
\text { propres intérêts. }\end{array}$ & $\begin{array}{c}2,10 \\
(0,90) \\
\end{array}$ & $\begin{array}{c}1,84 \\
(0,83) \\
\end{array}$ & $\begin{array}{c}1,92 \\
(0,86)\end{array}$ & $0,93^{* * *}$ \\
\hline 9. & $\begin{array}{l}\text { Je dis aux autres ce qu'ils } \\
\text { veulent bien entendre pour les } \\
\text { amener à faire ce que je veux. }\end{array}$ & $\begin{array}{c}1,79 \\
(0,84)\end{array}$ & $\begin{array}{l}1,42 \\
(0,69)\end{array}$ & $\begin{array}{l}1,54 \\
(0,76)\end{array}$ & $54,86^{* * *}$ \\
\hline 10. & $\begin{array}{l}\text { Ça me dérangerait que la } \\
\text { réussite me vienne aux dépens } \\
\text { d'un autre. }\end{array}$ & $\begin{array}{c}2,26 \\
(0,98)\end{array}$ & $\begin{array}{c}2,13 \\
(1,03)\end{array}$ & $\begin{array}{c}2,17 \\
(1,02)\end{array}$ & $0,95^{* * *}$ \\
\hline 11. & $\begin{array}{l}\text { Souvent, ça me plaît bien une } \\
\text { belle petite combine. }\end{array}$ & $\begin{array}{c}2,22 \\
(0,95)\end{array}$ & $\begin{array}{c}1,93 \\
(0,88)\end{array}$ & $\begin{array}{l}2,03 \\
(0,91)\end{array}$ & $17,52^{* * *}$ \\
\hline 12. & $\begin{array}{l}\text { Je me fais un point d'honneur } \\
\text { de ne pas blesser les autres dans } \\
\text { la poursuite de mes intérêts. }\end{array}$ & $\begin{array}{l}1,94 \\
(0,95)\end{array}$ & $\begin{array}{c}1,65 \\
(0,88)\end{array}$ & $\begin{array}{l}1,78 \\
(0,91)\end{array}$ & $0,57^{* * *}$ \\
\hline 13. & $\begin{array}{l}\text { Je prends plaisir à jouer avec les } \\
\text { sentiments des autres. }\end{array}$ & $\begin{array}{c}1,29 \\
(0,63) \\
\end{array}$ & $\begin{array}{c}1,17 \\
(0,51) \\
\end{array}$ & $\begin{array}{c}1,21 \\
(0,56) \\
\end{array}$ & $88,76^{* * *}$ \\
\hline 14. & $\begin{array}{l}\text { Je me sens mal si mes paroles } \\
\text { ou mes gestes font de la peine } \\
\text { à quelqu'un. }\end{array}$ & $\begin{array}{c}1,79 \\
(1,00)\end{array}$ & $\begin{array}{c}1,42 \\
(0,84)\end{array}$ & $\begin{array}{c}1,55 \\
(0,92)\end{array}$ & $77,38^{* * *}$ \\
\hline
\end{tabular}




\section{Annexe A (suite)}

\begin{tabular}{|c|c|c|c|c|c|}
\hline & Items & Hommes & Femmes & Total & $F$ \\
\hline 15. & $\begin{array}{l}\text { Même si j'essaie à tout prix de } \\
\text { vendre quelque chose, je n'irais } \\
\text { pas jusqu'à mentir pour le faire. }\end{array}$ & $\begin{array}{l}2,03 \\
(0,98)\end{array}$ & $\begin{array}{l}1,67 \\
(0,89)\end{array}$ & $\begin{array}{c}1,79 \\
(0,94)\end{array}$ & $4,11^{* * *}$ \\
\hline 16. & $\begin{array}{l}\text { Ce n'est pas bien de tricher } \\
\text { parce que c'est injuste pour } \\
\text { les autres. }\end{array}$ & $\begin{array}{c}1,93 \\
(0,93)\end{array}$ & $\begin{array}{l}1,69 \\
(0,89)\end{array}$ & $\begin{array}{c}1,77 \\
(0,91)\end{array}$ & $0,072^{* * *}$ \\
\hline 17. & $\begin{array}{l}\text { Je me retrouve devant le même } \\
\text { type de problèmes d'une fois à } \\
\text { l'autre. }\end{array}$ & $\begin{array}{c}2,17 \\
(0,85)\end{array}$ & $\begin{array}{c}2,25 \\
(0,84)\end{array}$ & $\begin{array}{c}2,23 \\
(0,85)\end{array}$ & $0,23^{* * *}$ \\
\hline 18. & J'éprouve souvent de l'ennui. & $\begin{array}{l}2,18 \\
(0,94)\end{array}$ & $\begin{array}{l}2,28 \\
(0,91)\end{array}$ & $\begin{array}{c}2,25 \\
(0,92)\end{array}$ & $0,39 * *$ \\
\hline 19. & $\begin{array}{l}\text { Je me sens capable de pour- } \\
\text { suivre un même but sur une } \\
\text { longue période de temps. }\end{array}$ & $\begin{array}{l}1,80 \\
(0,83)\end{array}$ & $\begin{array}{l}1,73 \\
(0,81)\end{array}$ & $\begin{array}{c}1,75 \\
(0,82)\end{array}$ & $0,029 * * *$ \\
\hline 20. & $\begin{array}{l}\text { Je ne fais pas de projets très } \\
\text { longtemps à l'avance. }\end{array}$ & $\begin{array}{c}2,41 \\
(0,95)\end{array}$ & $\begin{array}{c}2,23 \\
(0,93)\end{array}$ & $\begin{array}{c}2,29 \\
(0,94)\end{array}$ & $5,20 * * *$ \\
\hline 21. & $\begin{array}{l}\text { Je perds vite intérêt dans ce que } \\
\text { j'entreprends. }\end{array}$ & $\begin{array}{l}1,94 \\
(0,89)\end{array}$ & $\begin{array}{c}1,94 \\
(0,89)\end{array}$ & $\begin{array}{c}1,94 \\
(0,89)\end{array}$ & 0,005 \\
\hline 22. & $\begin{array}{l}\text { La plupart de mes problèmes } \\
\text { viennent du fait que les autres } \\
\text { ne me comprennent tout } \\
\text { simplement pas. }\end{array}$ & $\begin{array}{c}2,10 \\
(0,94)\end{array}$ & $\begin{array}{c}1,95 \\
(0,89)\end{array}$ & $\begin{array}{c}2,00 \\
(0,91)\end{array}$ & $4,35^{* * *}$ \\
\hline 23. & $\begin{array}{l}\text { Avant de faire quoi que ce soit, } \\
\text { j'en pèse toutes les consé- } \\
\text { quences possibles. }\end{array}$ & $\begin{array}{c}2,33 \\
(0,90)\end{array}$ & $\begin{array}{c}2,20 \\
(0,86)\end{array}$ & $\begin{array}{c}2,25 \\
(0,87)\end{array}$ & $11,43^{* * *}$ \\
\hline 24. & $\begin{array}{l}\text { J'ai eu un tas d'engueulades } \\
\text { avec d'autres personnes. }\end{array}$ & $\begin{array}{l}1,82 \\
(0,92)\end{array}$ & $\begin{array}{l}1,75 \\
(0,87)\end{array}$ & $\begin{array}{c}1,77 \\
(0,89)\end{array}$ & 3,44 \\
\hline 25. & $\begin{array}{l}\text { Quand je suis frustré(e), } \\
\text { souvent je me défoule en } \\
\text { piquant une crise de colère. }\end{array}$ & $\begin{array}{l}1,67 \\
(0,87)\end{array}$ & $\begin{array}{l}1,96 \\
(0,96)\end{array}$ & $\begin{array}{c}1,86 \\
(0,94)\end{array}$ & $4,36 * * *$ \\
\hline 26. & On surestime l'amour. & $\begin{array}{l}2,33 \\
(0,99)\end{array}$ & $\begin{array}{c}2,36 \\
(1,00)\end{array}$ & $\begin{array}{c}2,34 \\
(0,99)\end{array}$ & 0,45 \\
\hline
\end{tabular}

${ }^{*} p<0,05 ;{ }^{* *} p<0,01 ;{ }^{* *} p<0,001$. Levenson, M. R., Kiehl, K. A., \& Fitzpatrick, C. M. (1995). Traduction française par Sabourin, S., \& Lussier, Y. (1998).

ABStRACt - The Levenson Self-Reported Psychopathy Scale (LSRP; Levenson, Kiehl \& Fitzpatrick, 1995) is designed to assess subclinical psychopathic personality traits in the general population. In this measure, psychopathy is conceptualized along two distinct but interrelated factors, referred to as primary and secondary psychopathy. Several studies support the psychometric properties of the original version; however, none reported fidelity and convergent validity coefficients for the French adaptation of the LSRP. The current study aims to assess the factorial structure and convergent validity of the LSRP's French adaptation in young adults. A sample of 2529 French- 
Canadian participants, aged between 15 and 26 years old, completed the LSRP, the NEO-FFI, and answered questions on psychoactive substance use. Exploratory and confirmatory factor analyses supported the two-factor structure of the instrument. In addition, internal consistency coefficients from the original version were replicated. Measurement models for women and men were partially invariant, but men scored significantly higher than women on primary psychopathy and on the total scale. Finally, the LSRP showed good convergent validity coefficients. The French adaptation of the Levenson Self-Reported Psychopathy Scale shows good psychometric properties and results are similar to those obtained in the original version.

KEYWORDS - Psychopathy, validity, factor analyses, personality, psychoactive substances use.

RESUMEN - La escala auto-reportada de psicopatía de Levenson (LSRP; Levenson, et al., 1995) es un instrumento que pretende evaluar los rasgos de personalidad psicopáticos en el seno de la población general. Ésta conceptualiza la psicopatía en dos factores distintos, pero interrelacionados, llamados psicopatía primaria y secundaria. La validez del instrumento en su forma original está bien demostrada, pero ninguna información se encuentra disponible en lo que respecta a la adaptación francófona del instrumento. Por lo tanto, el estudio apunta a evaluar la estructura factorial y la validez convergente de la versión francesa en la población de adultos jóvenes. Una muestra de 2.529 participantes con edades comprendidas entre 15 y 26 años completan la escala auto-reportada de psicopatía, la NEO-FFI, y responden también a preguntas sobre las costumbres del consumo de sustancias psicoactivas. Los resultados de los análisis factoriales exploratorios y confirmatorios muestran que la adaptación francófona respeta la estructura en dos factores, con coeficientes de saturación y de consistencia interna para las dos escalas comparables a aquéllas de la versión original en inglés. Los modelos de medida para los hombres y las mujeres se han mostrado parcialmente invariables; los hombres obtienen resultados superiores a los de las mujeres en las escalas de psicopatía primaria y total. Finalmente, le LSRP demuestra buenos indices de validez convergente.

palabaras Clave - Psicopatía, validez, análisis factoriales, personalidad, consumo de sustancias psicoactivas. 